Daoistische Sukzession und ihre Legitimierung durch Ordination

Bumbacher, Stephan Peter

Posted at the Zurich Open Repository and Archive, University of Zurich

ZORA URL: https://doi.org/10.5167/uzh-144705

Book Section

Published Version

Originally published at:

Bumbacher, Stephan Peter (2017). Daoistische Sukzession und ihre Legitimierung durch Ordination. In: Renger, Almut-Barbara; Witte, Markus. Sukzession in Religionen : Autorisierung, Legitimierung, Wissenstransfer. Berlin: De Gruyter, 515-534. 


\title{
Daoistische Sukzession und ihre Legitimierung durch Ordination
}

\begin{abstract}
Daoism (aka Taoism), the indigenous Chinese religion, provides an interesting example of lineage succession and legitimation of religious status by means of ordination. Relying on much older ritual elements, succession is realized by a series of ritual steps. First, the master selects a suitable candidate among his disciples using tests that assess the moral qualities of his possible successor. Then both master and disciple swear an oath which binds both parties to the agreed obligations. The master transmits his religious texts together with their secret oral explanations to his successor. The disciple, in turn, bestows gifts upon his master. The most elaborate form of the ordination ritual which is to be found in the so-called lingbao tradition is discussed in detail.
\end{abstract}

\section{Der religiöse Kontext}

Das religiöse Feld des chinesischen Mittelalters (2. Jh. v. Chr.-7. Jh. n. Chr.) bestand im Wesentlichen aus vier ungleich großen Teilen. ${ }^{1}$ Am wichtigsten, weil omnipräsent, war der bis in früheste Zeiten belegte Ahnenkult. Die wichtigste soziale Einheit war die gemeinsame Abstammungslinie der männlichen Nachkommen desselben Gründungsahns. Auch wenn sich im Verlaufe der Zeit diese Linien in verschiedene Familien aufspalteten, wurden sie durch die Erinnerung an und den Kult für ihre gemeinsamen Ahnen zusammengehalten. Diesen gegenüber waren die Nachkommen letztlich verantwortlich. Ihr gutes Verhalten führte zu Wohlergehen der Ahnen, schlechtes Verhalten hatte "sozialen Abstieg« der Ahnen in ihrer eigenen Sphäre zur Folge. Entsprechend waren die Ahnen die primäre Belohnungs- und Bestrafungsinstanz. Sie mussten über alle wichtigen familiären Ereignisse rituell unterrichtet werden, damit sie ihre Nachkommen unterstützen konnten. Von ihnen erfuhr man zum Beispiel im Traum, ob sie sich etwa in einem nicht nach den geomantischen Regeln errichteten Grab unwohl fühlten. Dann bestraften sie die Nachkommen mit Krankheit. Die Lebenden opferten ihnen in do-ut-des-Manier, d.h. sie erwarteten Segnungen durch die Ahnen als Gegenleis-

1 Bumbacher, »Medieval Chinese Religions«. 
tung. Die Ahnen waren »verantwortlich« für die ökonomischen Bedingungen der Nachkommen und sorgten für deren sozialen Aufstieg (oder Abstieg).

An nächster Stelle sind die sogenannten lokalen Kulte zu nennen, also einer lokalen Gottheit gewidmete Heiligtümer, in denen sich zu bestimmten Zeiten Priester oder Kultverantwortliche sowie Gläubige einfanden, um Kulthandlungen $\mathrm{zu}$ vollziehen. Lokale Kulte konnten, wenn sie »erfolgreich« waren, d.h. wenn sie dafür bekannt waren, dass die Bitten der Gläubigen von der Gottheit erhört wurden, durch ein sich entwickelndes Pilgerwesen zu regionalen, unter Umständen gar zu gesamtchinesischen Kulten avancieren.

Als eigentliche chinesische »Hochreligion « kann man den Daoismus bezeichnen. ${ }^{2}$ Das Ziel der verschiedenen, sich ab dem 2. Jahrhundert n.Chr. entwickelnden daoistischen Traditionen bestand letztlich darin, körperlich unsterblich zu werden, wobei sie sich hinsichtlich der zu wählenden Praktiken zum Teil erheblich unterschieden. Auf die Gemeinschaft hin ausgerichteten Traditionen standen anachoretische und monastische gegenüber.

Den vierten Teil bildete der aus Indien stammende und ab etwa der Zeitenwende nach China einwandernde Buddhismus. Sein Erlösungsziel bestand im Erreichen des nirvana, des endgültigen Verlöschens aus dem unendlichen Kreislauf von Geburt, Leiden, Tod, Wiedergeburt (samsara). Zu erreichen suchte man es auf dem Wege der Entsagung und der Meditation, die zu einem Zustand der Erleuchtung führen konnte, in dem man sich der kosmischen wie menschlichen Gesetzmäßigkeit vollkommen bewusst wurde. Während der Jahrhunderte der Infiltration und Verbreitung in China veränderte sich der Buddhismus zwangsläufig. So musste er, zumindest im Laienbuddhismus, die Existenz einer Seele als »Trägerin « des karma akzeptieren, ${ }^{3}$ da in China die Vorstellung zweier Seelentypen ${ }^{4}$ im Menschen Allgemeingut war und die Grundlage des Ahnenkultes bildete, obwohl der Buddhismus im Ursprungsland die Doktrin des »Nichtvorhandenseins einer Seele« (Pāli anatta, Skt. anatman) in Abgrenzung zur ewig unveränderlichen Seele der brahmanischen Religion vertrat. Zudem verwendeten die Übersetzer der in indischen Sprachen verfassten Texte oft vermeintlich ähnliche daoistische Begriffe für buddhistische termini technici. Das hatte zur

2 Seidel, Taoismus.

3 Liebenthal, »Immortality of the Soul«; Pachow, »Immortality of the Soul«; Park, Buddhism. 4 Seit dem 4. Jahrhundert v.Chr. ist der chinesische Glaube an zwei verschiedene »Arten« von Seelen, die gleichzeitig den menschlichen Körper beleben, dokumentiert. Die hun- oder Hauchseele - zuständig für die »höheren« Eigenschaften des Menschen - verlässt den Körper nach dem Tode und wird zu einem Ahnen, die po- oder Körper-Seele, welche die elementaren Funktionen des Menschen kontrolliert, bleibt im Körper bis zum vollständigen Zerfall des Leichnams, dann tritt sie in die unterirdischen »Gelben Quellen« ein. 
Folge, dass den Chinesen die frühen Übersetzungen zwangsläufig wie daoistische Texte erschienen und man den Buddhismus als eine Spielart des Daoismus wahrnahm. Zudem wurde der Buddhismus tatsächlich insofern vom Daoismus beeinflusst, als er eine neue Textgattung hervorbrachte, die sogenannten apokryphen Schriften, welche sich zum Beispiel an den daoistischen apokalyptischen Texten orientierten. Vom 2. Jahrhundert n.Chr. an waren diese, welche Voraussagen von Katastrophenereignissen, Schilderungen bevorstehender gigantischer Überschwemmungen, eschatologischer Herrscher, göttlicher Retter (»Messias«-Gestalten), der eigentlichen Apokalypse, der Errettung weniger auserwählter Gläubiger und die Errichtung einer neuen kosmischen Ordnung zum Inhalt hatten, derart weit verbreitet, dass der Buddhismus mit eigenen Texten darauf reagieren musste. Der Buddhismus nahm nicht nur Elemente aus den lokalen Kulten und den verschiedenen daoistischen Traditionen auf, sondern beeinflusste auch die letzteren nachhaltig. ${ }^{5}$

\section{Frühe daoistische Sukzession}

Die möglicherweise früheste Beschreibung einer daoistischen Sukzession findet sich in Ge Hongs (283-343) Werk Baopuzi nei pian (»Meister, der die Einfachheit umfasst, die inneren Kapitel«). Danach war der Begründer der Tradition, welcher Ge Hong angehörte, Zuo Ci alias Zuo Yuanfang (um 200 n. Chr.). Von ihm heißt es:

Als sich in der Vergangenheit Zuo Yuanfang im Tianzhu-Gebirge der Meditation widmete, übergab ihm eine Gottheit die Schrift Jin dan xian jing (»Schrift von der Erlangung der Unsterblichkeit mittels Gold und Zinnober«). Da ihm, konfrontiert mit den Unruhen am Ende der Han-Dynastie, die Muße fehlte, [das Elixier] herzustellen, verließ er das Gebiet, überquerte den Yangtze [südwärts] und begab sich nach Osten, in der Absicht, sich auf einem bekannten Berg niederzulassen und diese Technik (nämlich die Alchemie) zu kultivieren. Mein Onkel [Ge] Xiangong war ebenfalls Schüler von [Zuo] Yuanfang und erhielt [den Text] von ihm. Insgesamt erhielt er das Taiqing dan jing (»Schrift vom Zinnober des Himmels der größten Reinheit») in drei Kapiteln, das Jiuding dan jing (»Schrift vom Zinnober der neun Kessel«) in einem Kapitel und das Jin yi dan jing (»Schrift vom verflüssigten Gold und dem Zinnober«) in einem Kapitel. Mein Lehrer, der Edle Zheng (Zheng Yin), war der Schüler meines Onkels Xiangong und erhielt sie von meinem Onkel. Da er aber von Hause aus arm war, hatte er nicht die Mittel dazu, die Ingredienzien [für die Elixiere] zu kaufen. Ich meinerseits diente ihm [als Schüler] und wusch und fegte [die Böden seines Hauses] über lange Zeit. Dann errichtete er auf dem Mazhi-Berg einen Altar und nach einer Eideszeremonie übergab er sie (die Schriften) [mir], ebenso die [geheimen] mündlichen Erläuterungen,

5 Mollier, Buddhism and Taoism. 
die zwar mündlich übermittelt, aber nicht schriftlich festgehalten [werden dürfen]. Vordem existierten diese Bücher nicht in Südchina. Sie wurden abgefasst von Zuo Yuanfang, Yuanfang überlieferte sie meinem Onkel, mein Onkel überlieferte sie dem Edlen Zhen und der Edle Zheng überlieferte sie mir. ${ }^{6}$

Dieser Bericht enthält bereits die typischen Merkmale der daoistischen Sukzession und Ordination: Ordination wurde in einem Ritual der Übergabe heiliger Schriften vollzogen. Am Anfang einer daoistischen Tradition oder Schule stand die Ordination des Schulbegründers nach bestandener Prüfung durch eine Gottheit. Die Sukzession setzte ein mit der Prüfung des geeigneten Schülers, der Übergabe der heiligen Schriften sowie der mündlichen Erläuterungen an ihn anlässlich einer feierlichen Eideszeremonie an einem Altar.

Die verschiedenen Elemente lassen sich auf ältere, vor-daoistische Ereignisse zurückführen. So findet sich zum Beispiel im historiographischen Werk Shi ji (»Aufzeichnungen des Historiographen«) des Sima Qian (ca. 145-86 v.Chr.) eine der frühesten Darstellungen der Übermittlung einer Schrift durch eine Gottheit an einen auserwählten Menschen. Kapitel 55 enthält die Vita des Zhang Liang, des Fürsten von Liu und vertrautesten Beraters des nachmaligen ersten Kaisers der Han-Dynastie, Han Gaozu.

\section{Eignungsprüfung}

In dieser Biographie findet sich eine längere Perikope, welche die Begegnung Zhang Liangs mit einem alten Mann auf einer Brücke schildert: ${ }^{7}$

Dieser ließ absichtlich seine Schuhe von der Brücke hinunterfallen, wandte den Kopf Liang zu und sagte zu ihm: »Hinunter, mein Söhnchen! Schuhe holen!« Liang, fassungslos [ob dieses Betragens], hätte jenen am liebsten verprügelt. Doch angesichts seines hohen Alters bezwang er sich, stieg hinab und holte die Schuhe. Sprach der Alte: »Zieh' mir die Schuhe an!« Da Liang ihm nun schon einmal die Schuhe geholt hatte, kniete er ehrerbietig nieder und zog sie ihm an. Der Alte nahm sie mit den Füßen entgegen und ging dann lachend davon. Über alle Maßen betroffen starrte Liang ihm nach. Der Alte hatte sich ungefähr eine Meile weit entfernt, da kam er wieder zurück und sprach: »Du bist schon einer Unterweisung würdig, mein Söhnchen. In fünf Tagen bei Sonnenaufgang komm hier mit mir zusammen!«

6 Baopuzi neipian jiaoshi 4,71; vgl. Ware, Nei P’ien, $69 \mathrm{f}$.

7 Shi ji 55,2034f. Das Folgende ist entnommen Bumbacher, »Quellenwert«, speziell 60-63. 
Ganz offensichtlich handelt es sich beim Verhalten des Alten um eine Prüfung der charakterlichen Eigenschaften des Zhang Liang. Dass er diese erste Prüfung bestand wird ersichtlich aus der Äußerung des Alten: »Du bist schon einer Unterweisung würdig, mein Söhnchen. «Doch wird er nochmals auf die Probe gestellt: Liang versprach in fünf Tagen wiederzukommen. Als er sich zur verabredeten Zeit auf der Brücke einfand, war der Alte bereits da und schalt Zhang Liang für sein Zuspätkommen. Er solle in fünf Tagen wieder kommen, dann aber früher! Liang erschien das nächste Mal früher, doch wiederholte sich dieselbe Szene. Nach weiteren fünf Tagen fand er sich bereits vor Mitternacht ein und war tatsächlich vor dem Alten da, der sich darüber freute, denn sein Schützling hatte nun auch diese Prüfung bestanden. Nun übergab er diesem eine Schrift offensichtlich göttlichen Ursprungs:

[Der Alte] zog ein Bündel beschriebener Holztäfelchen hervor und sprach: »Wenn du das liest, wirst du dereinst der Lehrmeister eines Königs werden. In zehn Jahren wird er auftreten. In dreizehn Jahren aber wirst du, mein Söhnchen, mich in Jibei sehen. Der gelbe Stein am Fuße des Gucheng Berges - der werde ich dann sein. « Dann ging er fort ohne ein weiteres Wort und ward nie wieder gesehen. Als der Morgen graute, nahm [Zhang Liang] dieses Buch in Augenschein, es war das Taigong bingfa (»Militärstrategie des Taigong«). Liang war darob erstaunt, doch lernte er beständig [darin] und sagte es laut vor sich her.

Dank dieser göttlichen Hilfe erwarb sich Zhang Liang außerordentliche strategische Fähigkeiten und wurde zum äußerst erfolgreichen Berater des späteren Kaisers Han Gaozu, der seinen Aufstieg zur Macht entscheidend ihm verdankte.

Die Ordination des daoistischen Schulgründers durch eine Gottheit dürfte im Fall des Ge Hong wohl in Meditation, genauer: in einer Visualisierung der Gottheit und deren Verkündigung der Schrift, erfolgt sein. ${ }^{8}$ Um die Sukzession zu sichern, setzte der Gründer einen seiner Schüler als Nachfolger und Überlieferer der Tradition ein, der seinerseits die Lehre an einen seiner eigenen Schüler weitergab.

Der Wahl des geeigneten Nachfolgers ging, wie im Beispiel des Zhang Liang, eine gründliche Prüfung des Schülers voraus. In Ge Hongs Beispiel ist sie angedeutet durch die Formulierung: »ich meinerseits diente ihm [als Schüler] und wusch und fegte [die Böden seines Hauses] über lange Zeit«. An anderer Stelle schildert Ge Hong dies ausführlicher:

8 Nebenbei bemerkt bedienten sich in China auch die Buddhisten dieser Methode, um neue (apokryphe) Texte, welche den veränderten Umständen Rechnung trugen, hervorzubringen. Vgl. Bumbacher, Empowered Writing, 136-139. 


\begin{abstract}
Alle anderen Schüler [des Zheng Yin] arbeiteten für ihn als persönliche Diener, sammelten Brennholz oder pflügten die Felder. Ich alleine - da ich schwächlich war und deren Arbeit nicht aushalten konnte und nichts besaß, womit ich selber körperliche Arbeit verrichten konnte - kehrte regelmäßig persönlich [die Böden], staubwischte die Betten und die Tische, zerrieb die Tusch[steine] und betreute die Lichtkerzen. ${ }^{9}$
\end{abstract}

Die Prüfungen beinhalteten also nicht das Abfragen von Wissen, sondern es wurden Motivation und charakterliche Eignung (Vertrauen in und Hingabe an den Meister, Egolosigkeit, Affektfreiheit, Demut) getestet. Auch von Gottheiten wird in verschiedenen daoistischen Texten gesagt, dass sie die Menschen prüften, bevor sie sich ihnen eröffneten. ${ }^{10}$

\title{
4 Eid als gegenseitige Verpflichtung von Meister und Schüler
}

Die Ordination bestand in der Übergabe der heiligen Bücher anlässlich einer Eideszeremonie. Wiederum in den Worten Ge Hongs:

Man verehrte diese Bücher (wörtlich: dieses Dao) und betrachtete sie als geheim. Und wer nicht dazu bestimmt war, unsterblich zu werden, konnte sie nicht erhalten. Man übermittelte sie nur einmal alle vierzig Jahre und übertrug sie, indem man den Mund mit Blut [eines Opfertieres] beschmierte, einen Eid schwor und Geschenke tauschend einen Bund schloss. ${ }^{11}$

Auf dem zuvor errichteten Altar wurde ein Opfertier geschlachtet, mit dessen Blut sich die Beteiligten, also der Lehrer und der in seiner Nachfolge stehende Schüler, den Mund bestrichen. Dadurch sollte zum Ausdruck gebracht werden, dass, wer sich später nicht an die Vereinbarungen des Eides hielt, sterben sollte. Als Zeugen und, im Falle von Zuwiderhandlungen gegen die Vereinbarung, als Vollstrecker der Strafe fungierten die angerufenen und am Altar präsenten Götter, an die das Tieropfer gerichtet war.

Dieses Ritual lässt sich auf zwei bedeutend ältere zurückführen, deren Kombination es darzustellen scheint, nämlich die Loyalitätseide einerseits und die Investitur der Lehensnehmer andererseits.

9 Baopuzi neipian jiaoshi 19,332; vgl. Ware, Nei P’ien, 311.

10 Für ein Beispiel, wie eine Göttin ihren menschlichen Schüler hinsichtlich seiner Standhaftigkeit bezüglich sexueller Askese prüft, siehe Bumbacher, Daoxue zhuan, 370.

11 Baopuzi neipian jiaoshi 19,336; vgl. Ware, Nei P’ien, 314. 
In der zweiten Hälfte des letzten Jahrhunderts kamen bei Ausgrabungen in Houma und Wenxian (Provinz Shanxi) beschriftete Jade- und Steintäfelchen zum Vorschein, die in die Zeit um 490 v.Chr. datiert werden und mit Eidestexten beschriftet sind. ${ }^{12}$ Darin schwören in der Regel Untergebene ihrem Herrn die Treue gegen dessen Widersacher. Sollten sie sich nicht daran halten, seien sie von den jenseitigen Mächten zu bestrafen. Der vollständig erhaltene Text 67,6 lautet: ${ }^{13}$

[Falls ich], NN, von diesem Tage an es wagen sollte, nicht respektvoll dem Wortlaut dieser Übereinkunft nachzukommen oder es noch in irgend einer Weise wagen sollte, die Angehörigen [der Widersacher meines Herrn] wieder einzusetzen, oder hören sollte, dass ältere oder jüngere Mitglieder meines Hauses in irgend einer Weise die Angehörigen [der Widersacher meines Herrn] wieder einsetzen, und [ich] es versäumen sollte, diese dingfest zu machen und [Deinem Recht] zu unterwerfen, mögen die ruhmreichen früheren Herzöge von Jin in ihren großen Gräbern weitsichtig mich augenblicklich entdecken und Ruin über mein Geschlecht bringen.

Dass der Beeidungsvorgang von einem Opfer begleitet war, geht aus (dem leider stark fragmentierten) Text 16,3 hervor:

Im elften Monat, am Tage jiayin, dem ersten Tag des Monats [beeidet]. Am Tage yichou werde ich es wagen, einen Ochsen zu schlachten [und mich zu wenden an] die glorreichen früheren (=verstorbenen) Fürsten von Jin [...]. [Falls ich nicht ...] mögen die früheren Fürsten [...] mich entdecken und Ruin [über mich und meine Nachkommen] bringen. ${ }^{14}$

Das die Vertragsabschlüsse begleitende Tieropfer stellt ein chinesisches Beispiel von longue durée dar. So kommentierte noch Kong Yingda (574-648) in seinem Subkommentar (shu) zum historiographischen Werk Zuo zhuan (»Überlieferungen des [Herrn] Zuo«; spätes 4. Jh. v.Chr.) den Eintrag »im dritten Monat [des ersten Jahres des Herzogs Yin von Lu (722 v.Chr.)] schlossen der Herzog [Yin] und Yifu von Zhu einen Vertrag in Mie« mit folgenden Worten:

Im Allgemeinen tötete man im Vertragsritual ein Opfer, beschmierte die Lippen mit dessen Blut und verkündete den weitsichtigen Geistern: »Falls irgendjemand [gegen diesen Vertrag] verstoßen oder ihn brechen sollte, sollen die Geister Unheil und Unglück auf ihn häufen und mit ihm so umgehen wie mit diesem Opfer[tier] umgegangen wurde. ${ }^{15}$

Das Ritual beschrieb Kong Yingda etwas detaillierter:

12 Weld, »Covenant Texts«.

13 Vgl. Weld, »Covenant Texts«, 149.

14 Vgl. Weld, »Covenant Texts«, $144 \mathrm{f}$.

15 Vgl. Weld, »Covenant Texts«, 156. 
Was nun den Vorgang des Vertragsschließens angeht, so hoben sie zuerst eine rechteckige Grube aus dem Boden, dann töteten sie das Opfer am Rande der Grube, schnitten das linke Ohr [des Tieres] ab, legten es in eine Perlenschale und ließen das Blut in eine Jade-Schüssel fließen. Dann verwendeten sie das Blut, um den Vertrag niederzuschreiben. Nach Beendigung des Schreibaktes beschmierten sie ihre Lippen mit dem Blut und verlasen den Text. ${ }^{16}$

Das zweite Ritual, welches in die Ordination der Daoisten integriert wurde, geht auf die Investitur der dem Kaiser untergebenen Prinzen zurück und ist in Texten der früheren Han-Dynastie (206 v.Chr.-5 n.Chr.) mehrfach erwähnt. Das (bis auf Zitate verschollene) Chunqiu da zhuan (»Große Überlieferung der Frühlinge und Herbste«) sagt:

Im Reich des Himmelssohnes (= Kaisers) gibt es einen großen Erdaltar. Seine nach Osten gerichtete [Seite] ist grün, die nach Süden gerichtete rot, die nach Westen gerichtete weiß, die nach Norden gerichtete schwarz und die nach oben gerichtete gelb. Schickte [der Himmelssohn] sich an, den Osten [des Reiches] als Lehen zu vergeben, so entnahm er grüne Erde [vom Altar], gab er den Süden als Lehen, entnahm er rote Erde, gab er den Westen als Lehen, so entnahm er weiße Erde, gab er den Norden als Lehen, so entnahm er schwarze Erde, gab er den oberen (=zentralen) [Bereich] als Lehen, entnahm er gelbe Erde. Für jede [Himmelsrichtung] entnahm er ein Objekt (=Erde) der ihr entsprechenden Farbe, wickelte sie in weißes mao-Gras und gab sie [dem Lehensnehmer], damit er daraus einen [eigenen] Erdaltar machen konnte. ${ }^{17}$

Drei solche Investituren sind denn auch in Sima Qians Shi ji erwähnt. Die erste lautet:

Am Tage yisi des vierten Monats des sechsten Jahres [der Regierungsdevise yuan zhou des Kaiser Han Wudi] (12. Juni 117 v.Chr.) veranlasste der Kaiser, dass der Chef-Zensor Tang im Ahnentempel inthronisiert wurde und er - [nämlich des Kaisers] Sohn Hong - als Prinz von Qi eingesetzt wurde. Er verkündete: »Oh, mein junger Sohn Hong, empfange diesen grünen Erdaltar[teil]. Wir haben ihn von den Ahnen geerbt und nachdem wir die alten [Gebräuche] geprüft haben, errichten wir für dich ein Lehensgebiet und geben Dir das östliche Gebiet $\mathrm{zu}$ Lehen. $^{18}$

Sowohl die Investitur von Lehensnehmern als auch die Beeidung von vertraglichen Vereinbarungen mittels Tieropfern erfolgte an entsprechenden Altären. In beiden Fällen waren es göttliche Wesen, einerseits die Ahnen der Jinherrscher, andererseits der Erdgott, die als Zeugen anwesend waren und als Vollstrecker von

16 Vgl. Weld, »Covenant Texts«, 157.

17 Shi ji 60,2115. Vgl. Chavannes, Le t'ai-chan, 452f.

18 Shi ji 60,2113. Vgl. Chavannes, Le t'ai-chan, 453. 
Sanktionen walteten, wenn etwa der Lehensnehmer sein Lehen nicht im Sinne des Herrschers verwaltete.

Der Daoismus in der Form, wie ihn Ge Hong kannte, griff auf das Eidesritual zurück, dadurch andeutend, dass der Schüler des Meisters geschworen haben dürfte, dass er die vom Meister erhaltenen heiligen Schriften und mündlichen Unterweisungen nur im Sinne des Meisters verwenden werde und dass ihm bei Zuwiderhandlung die Strafe des Altar-Gottes, des Zeugen und Vollstreckers, gewiss sein werde. Gleichzeitig handelte es sich bei diesem Ritual aber auch um eine Investitur, da der Schüler von seinem Lehrer als sein Nachfolger eingesetzt wurde, er also in der Sukzession des Letzteren stand.

\section{Der Meister als Garant und Übermittler des Wissens}

Einen wichtigen Aspekt stellt die Aussage dar, dass der Meister »sie (die Schriften) [mir] übergab, ebenso die [geheimen] mündlichen Erläuterungen, die zwar mündlich übermittelt, aber nicht schriftlich festgehalten [werden dürfen]«. Die heiligen Schriften des Daoismus stellten nur die eine Seite der Lehre dar, sie waren als solche nur beschränkt verständlich. Zum vollständigen Verständnis waren zusätzliche mündliche Angaben und Kommentare des Meisters unabdingbar. Dies macht Ge Hong auch an anderer Stelle deutlich:

Der Prinz von Huainan (Liu An, gest. 122 v.Chr.) kopierte [aus den Texten], um das Hungbao zhenzhong shu (»Kopfkissenbuch der vollkommenen Schätze«) zusammenzustellen. Obwohl dieser Text existiert, haben alle dessen wesentliche Inhalte geheim gehalten, notwendigerweise muss man die verborgenen Aspekte mündlich [weitergeben], der geschriebene Text ist zu erläutern, erst dann kann man [den Inhalt] realisieren. ${ }^{19}$

Der Schüler wird sich eidesstattlich insbesondere dazu verpflichtet haben, die mündlichen Auskünfte nur nach eingehender Prüfung des in Frage kommenden eigenen Schülers in der Zukunft weiterzugeben. Im Eid dürfte festgehalten worden sein, dass eine unkorrekte Weitergabe von Text und mündlichen Erläuterungen durch die Götter bestraft wird.

19 Baopuzi neipian jiaoshi 2,21f.; vgl. Ware, Nei P’ien, 51. 


\section{Die Gegengabe des Schülers}

Der Ordination ging, zumindest in der tianshi-, d.h. »Himmelsmeister«-Tradition, die Beschenkung des Meisters durch den Initianden voraus. Die Schriften, welche vom Meister auf den Schüler übertragen wurden, umfassten Register, welche zum Beispiel die Dämonen namentlich auflisteten, über die der Ordinierte nun gebieten konnte, oder welche die himmlischen Beamten aufführten, mit deren Hilfe er rechnen durfte. Die Ordination wird in einer säkularen Quelle, dem Sui shu (»[Dynastie-]Geschichte der Sui«, 636 n.Chr.) beschrieben:

[Von den Registern] gibt es viele, welche die Namen aller himmlischen Beamten und deren Assistenten beinhalten. [...] Diejenigen, welche sie erhalten, müssen sich zuerst mittels Fasten reinigen, dann beschaffen sie sich einen goldenen Ring sowie Seide als Geschenk für die Unterredung mit dem Meister. Nachdem dieser die Geschenke erhalten hat, übergibt er die Register. Dann spalten sie den goldenen Ring entzwei und beide nehmen eine Hälfte, von der sie sagen, sie sei ein Unterpfand [der Ordination]. ${ }^{20}$

Die Spaltung des Rings und Übergabe je einer Hälfte an je einen der beiden Beteiligten geht auf eine alte chinesische Tradition zurück, in der Objekte, zum Beispiel metallene Tigerfigürchen, geteilt wurden und zum Beispiel der Oberbefehlshaber die eine Hälfte und der ihm untergebene Offizier die andere als Legitimationszeichen behielt. Wenn der eine nun während einer Schlacht vom andern Hilfe anfordern wollte, musste sein Bote seine Hälfte dem anderen vorlegen. Beide Hälften wurden nun aneinandergelegt und nur, wenn sie perfekt passten, wurde der Bote und seine Botschaft als legitim angesehen. ${ }^{21}$ Möglicherweise mag die Teilung des Ringes noch daran angeknüpft und bedeutet haben, dass (in spirituellen Angelegenheiten) Meister und Schüler zu gegenseitiger Hilfeleistung verpflichtet waren.

\section{Legitimation durch den Himmel}

Ein vom bisher diskutierten Schema der daoistischen Ordination abweichendes Beispiel findet sich in der (nur fragmentarisch erhaltenen) Lebensbeschreibung der Daoistin Wang Daolian. ${ }^{22}$ Von ihr wird gesagt, dass sie sich durch Handel mit Textilien, Duftstoffen, Ölen und Nahrungsmitteln die finanziellen Grundlagen

20 Sui shu 35,1092. Vgl. Seidel, »Imperial Treasures«, 323f., sowie Ware, »Taoism«, 245.

21 Vgl. Bumbacher, Empowered Writing, 13-82.

22 Vgl. Bumbacher, Daoxue zhuan, 301-303. 
erarbeitete, welche es ihr erlaubten, ein Kloster zu errichten - aus traditionell konfuzianischer Sicht eine Unmöglichkeit, da Frauen keine eigenständigen Aktivitäten zugebilligt wurden. ${ }^{23}$ Weiter heißt es, dass die mehrgeschossigen Pavillons und die angereihten Wohnzellen, obwohl solide gebaut, in kurzer Zeit fertiggestellt waren, wie wenn göttliche Hilfe im Spiel gewesen sei. ${ }^{24}$ Danach ereigneten sich Wunder, worunter »sich eine jadene Schatulle befand, welche [vom Himmel] herabkam, sich auf den Altar niedersenkte und von einem Lichtschein umgeben war.« Dieses Behältnis enthielt offenbar eine Schrift, welche die Nonne in der Folge zehntausend Mal rezitierte, ${ }^{25}$ worauf »ein Wolkenwagen erschien, um sie abzuholen. Unter Donnergetöse und mächtigen Winden [wurde sie entrückt] und ein Wohlgeruch erfüllte den Himmel.« Ganz offensichtlich erregten die religiösen Aktivitäten der Wang Daolian die Zustimmung des Himmels, der sie durch die Herabsendung einer himmlischen Schrift legitimierte.

Die Beschreibung dieses Ereignisses lehnt sich an ein berühmtes, sehr altes Vorkommnis der chinesischen Geschichte an: die Legitimation der Eroberung der Shang-Dynastie (16. Jh. v.Chr.-1045 v.Chr.) durch die Zhou (1045 v.Chr.-250 v.Chr.). Als höchste religiöse Instanz wurde der Himmel (chin. tian) angenommen, der für die Belange des Staates maßgebend war. Er zeigte den Menschen, wenn ihm das Verhalten der Herrschenden missfiel. So diente die totale Mondfinsternis am 12. März des Jahres 1065 v.Chr. als ernste Warnung an die herrschenden Shang. Als sich deren Politik in der Folge nicht änderte, erschien fünf Jahre später gar ein Komet (der berühmte Halleysche Komet) und machte den Menschen der herrschenden Shang-Dynastie deutlich, dass das Ende ihrer Herrschaft unmittelbar bevorstand. Die an der Shang-Westgrenze siedelnde Ethnie der Zhou interpretierte das Zeichen in gleichem Sinne. Im Mai 1059 v.Chr. schließlich zeigte sich eine seltene Konjunktion der fünf sichtbaren Planeten im chinesischen Sternbild »Roter Vogel«, präziser: in der Gegend seines Schnabels. Da sich die Zhou traditionellerweise mit dem »Roten Vogel« identifizierten, interpretierten sie dieses Zeichen als eine Botschaft des Himmels, welche sie zur Machtübernahme aufforderte. ${ }^{26}$ Im späteren philosophischen Text Mo zi (»Meister Mo«) findet sich dieser Sachverhalt in einen Mythos gefasst, der Folgendes besagt:

23 Vgl. Bumbacher, "Abschied von Heim und Herd«.

24 Daoxue zhuan fr. 192 (Bumbacher, Daoxue zhuan, 301f.).

25 Über die Wirkungskraft von daoistischen (und buddhistischen) Schriften vgl. Bumbacher, Empowered Writing, 113-179.

26 Bumbacher, Empowered Writing, 38. 
Dann ließ sich ein roter Vogel mit einer Jadetafel im Schnabel auf dem Staatsaltar der Zhou am Berge Qi nieder. [Die Inschrift der Tafel] lautete: »Der Himmel befiehlt dem Zhou König Wen, die Yin (=Shang) anzugreifen und ihr Land in Besitz zu nehmen. $\ll^{27}$

In vergleichbarer Weise dürfte die Schrift in der Jade-Schatulle, die sich auf den Altar der Wang Daolian herabsenkte, im weitesten Sinne Weisungen des Himmels enthalten haben, deren Rezitation und Befolgung die Unsterblichkeit der Nonne Wang zur Folge hatte. Gleichzeitig, und wichtiger noch, stellte dieses Ereignis die Legitimierung all ihrer Taten durch die höchste religiöse Instanz, den Himmel dar. Diese war offenbar nötig, gerade weil Wang Daolian so offensichtlich entgegen den allgemein anerkannten (konfuzianischen) Normen weiblichen Verhaltens gehandelt hatte.

\section{Ordinationsritual der lingbao-Tradition}

Die ab dem 5. Jahrhundert gültige Form der Ordinationsliturgie wurde von $\mathrm{Lu}$ Xiujing (406-477) geschaffen. Als eine eminent wichtige Figur in der Geschichte des Daoismus ${ }^{28}$ machte er es sich einerseits zur Aufgabe, die echten Schriften der von Ge Chaofu (um 400 n.Chr.) begründeten lingbao-, d.h. »Wirkmächtige Juwelen«-Tradition von späteren Fälschungen zu trennen, zu katalogisieren (437), dadurch zu kanonisieren, und zu verbreiten. Andererseits präsentierte er im Jahre 471 den ersten Katalog der drei wichtigsten daoistischen Traditionen, das Sandong jingshu mulu (»Katalog der Schriften und Dokumente der drei Höhlen«), ${ }^{29}$ den er möglicherweise auf Geheiß des Kaisers zusammengestellt hatte, nachdem dieser das Chongxu-Kloster am Rande der Hauptstadt für ihn hatte erbauen lassen. Auf Lu Xiujing geht der um 454 fertiggestellte älteste in der daoistischen Sammlung Daozang erhaltene Text zurück, welcher von einer eigentlichen Ordinationsliturgie handelt, der Taishang dongxuan lingbao shoudu yi (»Zeremonien des Allerhöchsten für die Übergabe der lingbao[-Schriften] der Dongxuan [Abteilung des Daozang]«). Dabei griff Lu auf bestehende rituelle Elemente zurück, brachte sie in eine sinnvolle Abfolge und versah sie mit seinem Kommentar.

27 Mo zi 19,93. Vgl. Bumbacher, Empowered Writing, 38f.

28 Seine Biographie war im Daoxue zhuan enthalten, für die noch erhaltenen Fragmente siehe Bumbacher, Daoxue zhuan, 204-219.

29 Bokenkamp, »Lu Xiujing". 


\section{Zustimmung des Himmels}

Das lingbao-Ordinationsritual ist sehr komplex und erstreckt sich über eine Periode von mehreren Tagen. ${ }^{30}$ Auch hier sind die wesentlichen Elemente die Prüfung der zu Ordinierenden auf ihre Eignung hin, die Übergabe der heiligen Schriften sowie die Eideszeremonie. Freilich wurden diese nun rituell wesentlich erweitert. ${ }^{31}$ Zunächst war - ursprünglich unter freiem Himmel, nach Möglichkeit auf einem Berg - der Altar zu errichten:

[Die Beteiligten] haben eine hohe Plattform zu errichten, welche von einem Seil [als äußere Abgrenzung] umgeben ist, die fünf »Tore« aufweisen soll: nach Osten, nach Westen, nach Süden, nach Norden sowie in die königliche Richtung. ${ }^{32}$

Auf diesem Altar fand sodann die nächtliche Prüfung statt. Die zu übermittelnden Schriften und Dokumente wurden auf dem Altar offen ausgelegt und über Nacht liegen gelassen. Wenn sie durch den Wind durcheinandergewirbelt wurden, mussten sämtliche Beteiligten ein dreitägiges zhai-Ritual ${ }^{33}$ vollziehen und danach die Prüfungsprozedur wiederholen. Wenn nach drei solchen Prüfungsnächten die Schriftstücke jedes Mal vom Wind in Unordnung gebracht wurden, wurde das als Zeichen des Himmels gedeutet, der damit anzeigen wollte, dass der oder die Ordinationskandidat/in als nicht würdig zu erachten ist. ${ }^{34}$

\section{Ankündigung des bevorstehenden Rituals}

Der Priester musste nun offiziell die verschiedenen Gottheiten (des Himmels wie auch der zehn Himmelsrichtungen) davon unterrichten, dass die Ordinationszeremonie stattfinden werde, und sie als Zeugen einladen. $\mathrm{Zu}$ diesem Zwecke verfasste er eine schriftliche Petition, ${ }^{35}$ welche die wesentlichen Informationen

30 Eine umfassende Synopsis findet sich in Benn, Cavern-Mystery Transmisson, 121-136 und passim.

31 Wir werden uns aus Raumgründen nun auf diese beschränken, also die zentralen Elemente der Teile III, V und VI in der Zählweise Benns.

32 Taishang dongxuan lingbao shoudu yi (Daozang Nr. 528), 2b; vgl. Bokenkamp, »Early Lingbao Scriptures«, 110.

33 Hierbei handelt es sich im Wesentlichen um ein Reue-, Fasten- und Reinigungsritual. Vgl. Malek, Chai-chieh lu.

34 Taishang dongxuan lingbao shoudu yi $4 \mathrm{a}$.

$35 \mathrm{Zu}$ den Petitionen im Daoismus vgl. Bumbacher, Empowered Writing, 83-96. 
festhielt. Diese musste den Göttern zur Kenntnis gebracht werden. Das wurde dadurch realisiert, dass der Priester meditativ sogenannte Körpergottheiten ${ }^{36}$ aus seinem eigenen Körper hervorkommen und in den Himmel aufsteigen ließ, um das Dokument zu überbringen:

Respektvoll lasse ich aus meinem Körper die fünf perfekten Beamten der Gestalt (wu ti zhenguan) und die Verdienst-Gesandten (gong caoshi) hervorkommen und lasse die fliegenden Unsterblichen der drei Höhlen aus meinem Körper hervorkommen und aufsteigen zu den Drei Himmeln [um das Dokument zu übergeben]. ${ }^{37}$

Um sicherzustellen, dass die Petition auch tatsächlich allen bürokratischen Erfordernissen genügte, bat der Priester - vermittelt durch seine Körpergottheiten die zuständigen Beamten im Himmel, das Dokument zu überprüfen und gegebenenfalls Korrekturen darin vorzunehmen. Danach rief er seine Körpergottheiten wieder in seinen Körper zurück, wo sie für dessen korrektes Funktionieren und Schutz zuständig waren. ${ }^{38}$

Am Tage der Übergabe der Schriften informierte der Priester am Altar zunächst die in diesem Bereiche befindlichen Götter sowie diejenigen der Umgebung, der zehn Himmelsrichtungen und der heiligen Berge vom Vorhaben, so dass sie das Ritual überwachen und als Zeugen fungieren konnten. ${ }^{39}$ Dann entließ er aus seinem Körper Tausende von Körpergottheiten, sowohl zivile als auch bewaffnete, welche nunmehr die Aufgabe hatten, ihn und das Geschehen gegen übelwollende Dämonen und schlechte Einflüsse zu schützen. ${ }^{40}$

\section{1 Übergabe der heiligen Schriften und Legitimationszeichen}

Der wesentliche Schritt wurde eingeleitet durch die Ansage des Priesters, welche Schriften er nun dem/der Schüler/in ${ }^{41}$ unter Eid übergeben werde. Es schloss

$36 \mathrm{Zu}$ den Körpergottheiten siehe Bumbacher, »Körpergottheiten«.

37 Taishang dongxuan lingbao shoudu yi 4b. Vgl. Yamada, »Taoist Ritual«, 78.

38 Taishang dongxuan lingbao shoudu yi 7a. Vgl. Benn, Cavern-Mystery Transmission, 45.

39 Taishang dongxuan lingbao shoudu yi 8b.

40 Taishang dongxuan lingbao shoudu yi 13b-21a; vgl. Benn, Cavern-Mystery Transmission, 130. 41 Im Daoismus waren Frauen wie Männer weitgehend gleichberechtigt und konnten im Prinzip dieselben hierarchischen Positionen einnehmen - ganz im Gegensatz zum absolut patriarchalischen Konfuzianismus. Siehe Bumbacher, »Abschied von Heim und Herd«. 
sich das Öffnen der Schriftrollen und das Rezitieren ihres Inhaltes an, worauf die eigentliche Übergabe der Texte an den/die Schüler/in erfolgte. ${ }^{42}$ Letztere/r las den Eidestext des Priesters. ${ }^{43}$ Daraufhin erhielt der/die Initiand/in als äußeres Zeichen der Ordination drei hölzerne Täfelchen, etwa 43 Zentimeter lang und ca. 6 Zentimeter breit, die ba wei shen ce (»Göttlichen Täfelchen der acht Autoritäten«), welche er/sie von nun an am Gürtel zu tragen hatte. Sie enthielten die Namen des Meisters und des Schülers, trugen noch zusätzliche kurze Aufschriften und dienten als Talismane. ${ }^{44}$ Ihre Funktion war somit eine doppelte: einerseits sichtbares Zeichen der Legitimation durch den Meister, andererseits apotropäisches Hilfsmittel gegen wilde Tiere und Dämonen. ${ }^{45}$

Als weithin sichtbares Zeichen erhielt der/die Initiand/in nun den PriesterStab. Wie in den ursprünglichen lingbao-Texten beschrieben war dies ein über zwei Meter langer Bambusstock mit sieben Knoten zwischen den jeweiligen Segmenten. ${ }^{46}$ Der Schüler hatte nun die von den höchsten Göttern entsandten und den Stab unsichtbar umgebenden und beschützenden fünf männlichen und fünf weiblichen Gottheiten zu visualisieren. Dann erhielt er vom Meister nacheinander fünf beschriftete Talismane und musste $\mathrm{zu}$ jedem den geheimen Namen der damit verbundenen Gottheit aussprechen und dann ein qi (»Energie-Wolke«) in der entsprechenden Farbe visualisieren, welches durch seinen Mund in seinen Körper eintrat und sich in das entsprechende Körperorgan ergoss. Der Meister führte danach in jede der fünf zuvor angebrachten Öffnungen in den Knoten des Stabes jeweils einen der Talismane ein und verschloss diesen. Schließlich versiegelte der Meister die beiden Enden des Stabes. ${ }^{47}$ Durch dieses Ritual wurde eine enge Verbindung zwischen den Gottheiten, den Energien, aus denen sie letztlich bestanden, den von ihnen autorisierten Talismanen, dem Stab und schließlich dem Körper des Adepten hergestellt.

42 Taishang dongxuan lingbao shoudu yi 25b.

43 Taishang dongxuan lingbao shoudu yi 31b.

44 Taishang dongxuan lingbao shoudu yi 31b. Vgl. Bokenkamp, »Early Lingbao Scriptures«, 106, der eine ausführlichere Beschreibung aus einem der originalen lingbao-Schriften liefert.

45 Vgl. Bumbacher, Empowered Writing, 13-82.

46 Bokenkamp, »Early Lingbao Scriptures«, 104.

47 Taishang dongxuan lingbao shoudu yi 33a-35b. Vgl. Bokenkamp, »Early Lingbao Scriptures«, 104-106; Benn, Cavern-Mystery Transmission, 57-60. 


\section{Dokumentierung der Sukzessionslinie}

Bevor es nun zur eigentlich Eideszeremonie kam, welche die wechselseitige Bindung von Meister und Schüler zur lingbao-Tradition bekräftigte, rezitierte der Meister die »Genealogie« der übermittelten lingbao-Schriften: Letztlich entstammten sie demselben Ur-qi (Ur-»Energie«) wie die höchste Gottheit und wie schließlich der ganze daraus hervorgegangene Kosmos. ${ }^{48}$ Die höchste Gottheit Yuanshi tianzun (»Himmlischer Erhabener des Ur-Anbeginns«) verbreitete die wahren Texte (im Himmel), wo sie von verschiedenen Gottheiten ihrem absteigendem Range nach nacheinander kopiert wurden, bis sie schließlich über Ge Xuan der irdischen Welt bekannt gemacht und seinen Schülern überliefert wurden. Diese gaben sie ihrerseits an ihre eigenen Schüler weiter, bis zum Lehrer des aktuellen Meisters, der sie jetzt schließlich seinen Adepten im aktuellen Ordinationsritual übergab.

\section{Vereidigung}

So wie der Meister seinem Lehrer gegenüber durch einen mit der Übergabe geleisteten Eid verbunden war, so musste nun der gegenwärtige Adept nach dem Empfang dieser Schriften seinem Meister gegenüber einen Eid leisten. Auf die Ermahnung des Meisters - »entsprechend den ming ke (`leuchtenden Statuten «) sollst Du Dir gegenüber Selbstrespekt hegen und Dich selber ermutigen, rein und aufrichtig zu sein, Dich arbeitsam abzumühen, die weltlichen Verstrickungen zu unterbinden und Dein Sinnen ausschließlich auf [die Verbreitung] des Großen Gefährts [unserer Lehre] zu richten ${ }^{49}$ - leistete der frisch Ordinierte nun den Eid:

[...] Entsprechend werde ich mich selber ermutigen und [meine] Person respektieren, werde [den Gottheiten] Opfer darbringen und die Rituale ehren, werde die Affekte aufgeben und [mich] von den Bindungen [an die Welt] lösen, werde [mein] Streben nach abgeschiedenen Bergen ausrichten, werde [mich] für immer vom Staub der Welt abwenden, werde [mein] Sinnen ausschließlich nach Hügeln und Klippen richten, werde Tag und Nacht [mich] der Verehrung [der Götter] widmen, werde [mich] auf das Loswerden [meiner] einfältigen und verengten [Sehweisen] konzentrieren, werde [mich] gänzlich auf die Schriften der Weisen stützen, werde die vermodernden Knochen [der Menschen] herausgreifen und zur Erlösung führen, werde mit ganzem Herzen aufrichtig sein, und es wird nicht vorkommen, dass [ich]

48 Dieser erste Schritt steht nicht in diesem Text und muss basierend auf originalen lingbaoSchriften ergänzt hier intrapoliert werden. Vgl. Bumbacher, »Cosmic Scripts«.

49 Taishang dongxuan lingbao shoudu yi 36b, vgl. Bokenkamp, »Early Lingbao Scriptures«, 122. 


\begin{abstract}
Hintergedanken habe. Ich werde es nicht wagen, dem Eid zuwider zu handeln und [mich] vom Schwur abzuwenden, meinen Lehrer zu hintergehen oder das Dao (das universelle Gesetz) zu missachten. Sollte [ich zwar den richtigen] Anschein erwecken, [aber] nicht die [richtige] Gesinnung haben, werde ich [von den Göttern] nicht beschützt werden während der Zahl der [künftigen] Äonen ${ }^{50}$. [... $]^{51}$
\end{abstract}

Nach einer Reihe weiterer Zeremonien las der Priester die Beichte vor, in der er um Vergebung für seine und seiner Schüler Vergehen und Zuwiderhandlungen gegen die Gebote bat. Im anschließenden Gebet ersuchte er um die Erfüllung der Ziele des Kaisers, des Volkes, der Geister und der Götter. ${ }^{52}$ Schlussendlich wurde ein dreitägiges zhai-Ritual zum Dank für die gewährte Gnade durchgeführt. ${ }^{53}$

\title{
14 Von der »individuellen« zur »universellen« Ordination
}

Was zur Zeit des Ge Hong - und vielleicht auch bei Lu Xiujing - noch eine gleichsam intime Angelegenheit gewesen sein mochte und sich zwischen Meister, Schülern und Altar- bzw. Erdgottheiten abgespielt hatte, wurde im Laufe der Zeit zu einer Angelegenheit »kosmischen Ausmaßes«, da auch die höchsten Götter des Himmels, die Götter der Himmelsrichtungen, die lokalen und Naturgötter sowie die Körpergottheiten involviert wurden. Den vorgängigen Eignungsprüfungen durch den Meister folgte nun die »Befragung« des Himmels über die Eignung des/der zu Ordinierenden mittels der nächtlich ausgelegten Schriften. Das zeigt den erweiterten Anspruch der Daoisten, nicht mehr nur im Lokalen verwurzelt $\mathrm{zu}$ sein, sondern gewissermaßen als Interpreten und Vollstrecker »kosmischen« Willens aufzutreten, womit sie eigenem Ansprüche nach auch über dem Kaiserhaus standen und $\mathrm{zu}$ dessen religiöser Legitimation beitragen konnten. ${ }^{54}$ Die Legitimation der daoistischen Sukzession wurde so durch das gesamte Universum gewährleistet.

50 Mit dem Wort jie (wörtlich »plündern«, »rauben«), hier mir »Äonen« übersetzt, wird das Sanskritwort kalpa wiedergegeben, das über den Buddhismus Eingang in die chinesische Vorstellungswelt gefunden hat. Dies ist ein Hinweis auf die (wechselseitige) Beeinflussung von Buddhismus und Daoismus in China. Vgl. z.B. Zürcher, »Buddhist Influence«.

51 Taishang dongxuan lingbao shoudu yi 37b; vgl. Bokenkamp, »Early Lingbao Scriptures«, 122.

52 Taishang dongxuan lingbao shoudu yi 43b-46a; vgl. Benn, Cavern-Mystery Transmission, 134.

53 Taishang dongxuan lingbao shoudu yi $50 \mathrm{~b}$.

54 Benn, »Taoism as Ideology«. 
Auch die Anzahl der irdischen Direktbeteiligten vergrößerte sich entsprechend: Zum Meister als die Ordination vollziehendem Priester gesellten sich weitere Ritenspezialisten, insbesondere auch Kantoren und Riten-»Bedienstete« hinzu. Schließlich waren auch Verwandte und Freunde der zu Ordinierenden zugelassen, welche mitgeholfen hatten, das (teure) Eidesunterpfand zu finanzieren.

Der/die Ordinierte stand in der Sukzession seines Meisters, der seinerseits die Nachfolge seines Meisters angetreten hatte usw. bis schließlich zurück zu der Gottheit, die am Ursprung der durch sie begründeten Tradition die Schriften dem ersten irdischen Glied der Sukzessionskette übermittelt hatte. Das die Sukzessionsgenerationen verbindende Element waren die in der Ordination zu übermittelnden heiligen Schriften sowie die zusätzlich notwendigen mündlichen Informationen. Gewährleistet wurde die Legitimation einerseits durch die bestandenen Eignungsprüfungen, andererseits durch die Zeugenschaft der Götterwelt, welche die Einhaltung der Eide überwachte und bei Zuwiderhandlung sanktionierte. 


\section{Literatur}

Baopuzi neipian jiaoshi, hg. von Wang Ming, Peking: Zhonghua shuju, 1985.

Benn, Charles D., »Taoism as Ideology in the Reign of Emperor Hsüan-tsung (712-755)«, Diss., University of Michigan, 1977.

Benn, Charles D., The Cavern-Mystery Transmission. A Taoist Ordination rite of A.D. 711, Honolulu: University of Hawaii Press, 1991.

Bokenkamp, Stephen R., »Lu Xiujing, Buddhism, and the First Daoist Canon«, in: Scott Pearce u. a. (Hg.), Culture and Power in the Reconstitution of the Chinese Realm, 200-600, Cambridge, Mass.: Harvard University Press, 2001, 181-199.

Bokenkamp, Stephen R., »The Early Lingbao Scriptures and the Origins of Daoist Monasticism «, Cahiers d'Extrême Asie 20 (2011), 95-124.

Bumbacher, Stephan Peter, »Abschied von Heim und Herd. Die Frau im mittelalterlichen Daoismus und Buddhismus «, Asiatische Studien / Etudes Asiatiques 52 (1998), 673-694.

Bumbacher, Stephan Peter, "Cosmic Scripts and Heavenly Scriptures. The Holy Nature of Taoist Texts«, COSMOS, The Journal of the Traditional Cosmology Society 11,2 (1995), 139-153.

Bumbacher, Stephan Peter, Empowered Writing. Exorcistic and Apotropaic Rituals in Medieval China, St. Petersburg, Fla.: Three Pines Press, 2012.

Bumbacher, Stephan Peter, »Medieval Chinese Religions. An Example of /Selective Participation «, in: Christine Lienemann-Perrin und Wolfgang Lienemann (Hg.), Religiöse Grenzüberschreitungen. Studien zu Bekehrung, Konfessions- und Religionswechsel, Wiesbaden: Harrassowitz, 2012, 611-631.

Bumbacher, Stephan Peter, The Fragments of the Daoxue zhuan. Critical Edition, Translation and Analysis of a Medieval Collection of Daoist Biographies, Frankfurt am Main: Lang, 2000.

Bumbacher, Stephan Peter, „Zu den Körpergottheiten im chinesischen Taoismus«, in: Axel Michaels, Daria Pezzoli-Olgiati und Fritz Stolz (Hg.), Noch eine Chance für die Religionsphänomenologie?, Frankfurt am Main: Lang, 2001, 151-172.

Bumbacher, Stephan Peter, „Zum religionsgeschichtlichen Quellenwert des literarischen Genres zhuan (`Lebensbeschreibung») in Daoismus und chinesischem Buddhismus", in: Peter Schalk (Hg.), Geschichten und Geschichte. Historiographie und Hagiographie in der asiatischen Religionsgeschichte, Uppsala: Uppsala Universitet, 2010, 57-95.

Chavannes, Edouard, Le t'ai-chan. Essai de monographie d'un culte chinois, Paris: Leroux, 1910.

Liebenthal, Walter, »The Immortality of the Soul in Chinese Thought«, Monumenta Nipponica 8 (1952), 327-397.

Malek, Roman, Das Chai-chieh lu. Materialien zur Liturgie im Taoismus, Frankfurt am Main: Lang, 1985.

Mollier, Christine, Buddhism and Taoism Face to Face. Scripture, Ritual, and Iconographic Exchange in Medieval China, Honolulu: University of Hawaii Press, 2008.

Mo zi, Bd. 6, Taipeh: Xin bian zhu zi ji cheng, 1974.

Pachow, W., "The Controversy over the Immortality of the Soul in Chinese Buddhism «, Journal of Oriental Studies 16 (1978), 21-38.

Park, Jungnok, How Buddhism Acquired a Soul on the Way to China, Oxford: Equinox, 2012.

Seidel, Anna, »Imperial Treasures and Taoist Sacraments, Taoist Roots in the Apocrypha«, in: Michel Strickmann (Hg.), Tantric and Taoist Studies in Honour of R.A. Stein (Mélanges 
chinois et bouddhiques 21), Brüssel: Institut Belge des Hautes Etudes Chinoises, 1983, 291-371.

Seidel, Anna, Taoismus, die inoffizielle Hochreligion Chinas, Tokio: Deutsche Gesellschaft für Natur- und Völkerkunde Ostasiens, 1990.

Shi ji, Peking: Zhonghua shuju, 1982.

Sui shu, Peking: Zhonghua shuju, 1973.

Ware, James R., The Nei P'ien of Ko Hung. Alchemy, Medicine, and Religion in the China of A.D. 320, Cambridge, Mass.: M.I.T. Press, 1966.

Ware, James R., »The Wei shu and the Sui shu on Taoism «, Journal of the American Oriental Society 53 (1933), 217-249.

Weld, Susan R., »The Covenant Texts from Houma and Wenxian «, in: Edward L. Shaughnessy (Hg.), New Sources of Early Chinese History. An Introduction to the Reading of Inscriptions and Manuscripts, Berkeley: Institute of East Asian Studies, 1997, 125-160.

Yamada Toshiaki, »The Evolution of Taoist Ritual. K'ou Ch'ein-chih and Lu Hsiu-ching «, Acta Asiatica 68 (1995), 69-83.

Zhengtong Daozang, von 1444 mit Supplement von 1607, 1487 Texte, photomechanische Reproduktion in 1120 Faszikeln gebunden, Shanghai: Commercial Press, 1926.

Zürcher, Eric, »Buddhist Influence on Early Taoism. A Survey of Scriptural Evidence«, T'oung Pao 66 (1980), 84-147. 